\title{
Prognostic Value of Initial Glasgow Coma Score in Comatosed Patients on medical floor
}

\author{
M A NADEEM K IRFAN K A I WAHEED \\ Department of Medicine, King Edward Medical University/Mayo Hospital, Lahore \\ Correspondence to Dr: Muhammad Arif Nadeem Assistant Professor. Phone: 03008477287
}

Objective: An open non-randomized prospective observational study of comatosed patients was conducted to correlate initial Glasgow Coma Score (GCS) with the outcome in patients having medical coma at Mayo Hospital, Lahore between January 2002 to September 2003. Methods: A total of 273 patients were followed throughout their hospital stay to determine the outcome after the current episode of coma. A thorough history and examination was obtained for each case. The 3 category Glasgow Coma Score was recorded as an indication of the degree of unconsciousness. One of three possible outcome categories was established, recovery (discharge), death, and referral or self discharge (by attendants) against medical advice. Results: The outcome of 248 patients $(90.84 \%)$ was established i.e. $152(61.29 \%)$ were discharged after recovery and $96(38.71 \%)$ died. The remaining $25(09.16 \%)$ patients were lost to follow up. Four subcategories of the Glasgow Coma Score were made, at 3-point intervals. In the lowest score category (score 3-5) there was $59.67 \%$ mortality and only $30.66 \%$ patients were successfully treated and discharged. In the category of GCS $6-8,30.91 \%$ died and $60 \%$ were discharged, while in the $9-11$ category, $26.47 \%$ died and $66.18 \%$ were discharged. In the highest score category (12-15), $27.27 \%$ deaths occurred, probably related to the severity of main event, and $62.50 \%$ were discharged. Conclusion: The depth of coma as reflected by the initial GCS can reliably be used to predict the outcome in comatosed patients.

Key words: Glasgow Coma Score, correlation, outcome, coma, initial score.

Coma and the degree of altered consciousness have no significance as far as the nature of the underlying pathogenic process is concerned. It is useful to assign a quantitative term to the degree of impairment. This enables the physician to monitor response to therapy as well as the progression of the disease process and the prognostic significance of the degree of coma at presentation'. To note the degree of coma, one can use descriptive nomenclature like hypersomnolence, stupor, delirium or coma, which has the merits of brevity, with some loss of precision. A balance between these extremes has been struck by devising scoring systems, which quantify the patient's responses to yield a numerical value for the degree of coma. Many such scoring systems like the Edinburgh Scale have been devised ${ }^{2}$. The most widely used scoring system with a wealth of research background is the Glasgow Coma Scale (GCS) ${ }^{3.4,5}$.

The Edinburgh Scale (table 1) remains the favorite of resident staff around the world due to its simplicity ${ }^{2}$. The Glasgow Coma Scale is a numerical system, which was originally developed to determine the degree of coma in patients with head injury, its use was later extended to include patients with Medical coma as well (table 2) ${ }^{3,4,5}$. It has been used to guide initial management, predict outcome and as a standard against which newer investigations and therapies can be assessed ${ }^{6}$.

In Barsic et al.'s study, the prognostic significance of the day-1 GCS was evaluated in patients in an infectious diseases ICU, with the conclusion that while a significant relationship exists between the first day GCS and the subsequent ICU mortality in patients with CNS infections, such a correlation is not seen in patients with non-CNS infections leading to $\mathrm{coma}^{7}$. Another ethically important utility of the Glasgow Coma Scale was evaluated in a large study of comatose cardiac arrest survivors, using both coma scales and several of their constituent variables, as early as third day post-arrest, it was possible to identify with certainty those patients who were likely to remain permanently comatose or severely disabled. The best predictor was absence of motor response to pain. Thus the issue of terminati on of care in post-cardiac arrest patients can be decided as early as the third day, without compromising ethical criteria ${ }^{8}$.

Many other scoring systems have been devised for use in special situations. These incorporate neurological assessment as the major criterion but also include measurements pertaining to other vital functions as well. These include the APACHE II and III scoring system (Acute Physiology And Chronic Health Evaluation) used in intensive care patients, the Japan Coma Scale (10 grade); the ten category Adelaide Scale; a 13 category Jacobi Scale; SAPS (Simplified Acute Physiology Score); the OSF (Organ System Failure score) and the Swedish Reaction Level Scale ${ }^{9,}{ }^{10}$. In particular the APACHE II system has had the benefit of numerous trials, proving its utility in stratifying prognosis of critically ill patients. ${ }^{11}$ Studies have been undertaken to determine the interobserver variability of these scales and their comparison with Glasgow Coma Scale ${ }^{12-13}$. Most have found these systems comparable or inferior to Glasgow Coma Scale in the ease of use, reproducibility, lack of ambiguities and prognostic significance. Thus one may conclude that the use of special scoring systems should be judiciously infrequent, considering that none has been shown to be superior to the time-tested Glasgow Coma Scale. 
Aims and objectives: To correlate Initial Glasgow Coma Score with the Outcome in Patients having Medical Coma.

Material and methods: This was an open non-randomized prospective, observational study in which the participants were followed through their hospital stay. Patients were admitted through emergency. A total of 273 patients presenting in emergency ward with sustained impairment of consciousness (for more than one hour) were studied.

Inclusion criteria: Sustained impairment of consciousness occurring in patients older than 12 years of age due to causes other than trauma.

\section{Exclusion criteria}

- Brief (episodic) impairments of consciousness lasting less than one hour, thereby falling under the definition of syncope.

- Traumatic coma.

- Patients falling in paediatric age group.

- Patients succumbing to their illness so rapidly that any diagnostic work up could not be performed.

Methods: A thorough history and examination was obtained for each case. The findings were then subjected to statistical analysis. All the patients were managed intensively, and as far as possible using available resources, optimally, during their hospital stay. The 3 category Glasgow Coma Score was recorded as an indication of the degree of unconsciousness. The patients were followed through their hospital stay to determine the outcome after the current episode of coma. One of three possible outcome categories was established, recovery (discharge after they had gained consciousness with or without residual deficit), death, and a category in which the proximate outcome remained unknown because they could not be followed up further, either due to referral, or self discharge against medical advice.

\section{Results:}

In this study there were 156 male and 117 female patients, making up $57.14 \%$ and $42.86 \%$ of the cases respectively. Coma due to metabolic causes was the largest category in our study, making up about $54 \%$ of all causes. The second largest category was structural coma (29\%), followed by diffuse cerebral pathology causing coma (13\%). Psychogenic unresponsiveness (conversion or malingering), $(2 \%)$ completed the tally of diagnosed cases, whereas almost $2 \%$ of cases remained undiagnosed. The outcome of 248 patients $(90.84 \%)$ with medical coma was established in the study. The remaining 25 patients were lost to follow up either because of referred to a specialist unit (7 cases) or were discharged on request or left against medical advice (a common problem in our part of Sub-continent) i.e. 18 patients. Out of these patients, $152(61.29 \%)$ were discharged after recovery and $96(38.71 \%)$ died.

In our study, it was attempted to find out if there was any relationship between the depth of coma at presentation, as reflected by the initial Glasgow Coma Score and the outcome. Four subcategories of the Glasgow Coma Score were made, at 3-point intervals. A significant relationship between the initial Glasgow Coma Score and outcome was demonstrated (table 3 \& fig.1). It was found that in the lowest score category (score 3-5) there was $59.67 \%$ mortality and only $30.66 \%$ patients were successfully treated and discharged. In the category of GCS 6-8, $30.91 \%$ died and $60 \%$ were discharged, while in the $9-11$ category, $26.47 \%$ died and $66.18 \%$ were discharged. In the highest score category (12-15), $27.27 \%$ deaths occurred, probably related to the severity of main event, and $62.50 \%$ were discharged.

Fig. 1 Glasgow coma score with outcome

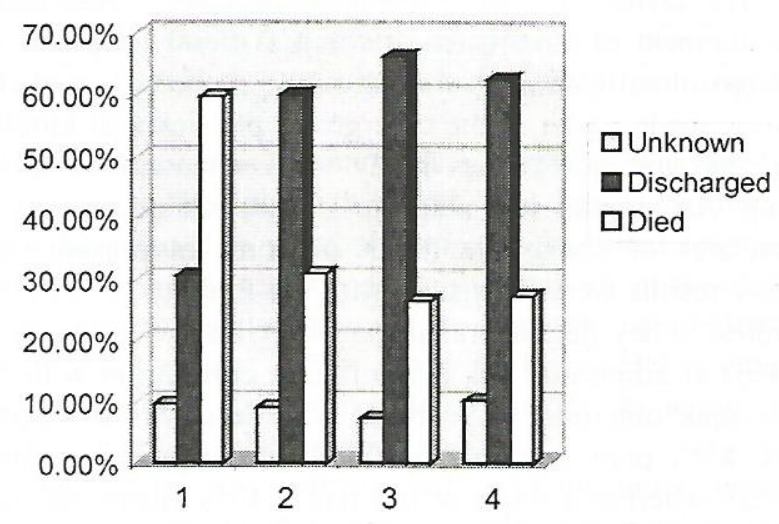

Table 1: The Edinburgh Scale

\begin{tabular}{ll}
\hline Coma grade $\mathrm{O}$ & Fully conscious \\
Coma grade 1 & Drowsy but responsive to vocal commands \\
Coma grade 2 & $\begin{array}{l}\text { Unconscious but responsive to minimal } \\
\text { painful stimuli }\end{array}$ \\
Coma grade 3 & $\begin{array}{l}\text { Unconscious but just responsive to strong } \\
\text { painful stimuli }\end{array}$ \\
Coma grade 4 & Unconscious with no response to stimuli \\
\hline
\end{tabular}

Table 2: The Glasgow Coma Scale

\begin{tabular}{ll}
\hline Eye opening (E) & 4 \\
Spontaneous & 3 \\
To speech & 2 \\
To pain & 1 \\
Nil & \\
Best Motor Response (M) & 6 \\
Obeys & 5 \\
Localizes & 4 \\
Withdraws & 3 \\
Abnormal flexion & 2 \\
Extensor response & 1 \\
Nil & \\
Best Verbal Response (V) & 5 \\
Oriented & 4 \\
Confused conversation & 3 \\
Inappropriate words & 2 \\
Incomprehensive sounds & 1 \\
Nil &
\end{tabular}

Glasgow Coma Score $=\mathrm{E}+\mathrm{M}+\mathrm{V}$ 
Table 3: Correlation of (Glasgow coma score with outcome

\begin{tabular}{|c|c|c|c|c|c|c|c|c|}
\hline \multirow[t]{3}{*}{ Outcome } & \multicolumn{8}{|c|}{ Glasgow Coma Score } \\
\hline & \multicolumn{2}{|c|}{$3-5$} & \multicolumn{2}{|l|}{68} & \multicolumn{2}{|c|}{$9-11$} & \multicolumn{2}{|c|}{$12-15$} \\
\hline & No. & $\%$ & No. & $\%$ & No. & $\%$ & No. & $\%$ \\
\hline Died & 37 & 59.67 & 17 & 30.91 & 18 & 26.47 & 24 & 27.27 \\
\hline Discharged & 19 & 30.66 & 33 & 60.00 & 45 & 66.18 & 55 & 62.50 \\
\hline Unknown & 06 & 09.67 & 05 & 09.09 & 05 & 07.35 & 09 & \\
\hline Total & 62 & & 55 & & 68 & & 88 & \\
\hline
\end{tabular}

\section{Discussion:}

Coma, a problem of overwhelming importance in any medical setup, has been the subject of many studies from a prognostic point of view ${ }^{14,15,16}$. Only a few published studies on the etiology of coma in Pakistan have been done and the significance of these studies lies in the fact that knowledge of the common causes of coma is a crucial time saving factor in the management ${ }^{16,}{ }^{17}$. Successful management of a comatose patient critically depends on prompt identification of the causative pathology, and this task is made easier if the emergency physician is familiar with the common causes likely to be encountered in his setup. An attempt was made to study possible prognostic correlates of coma like depth of coma at presentation. These results were consistent with the findings of previous studies $^{6}$. They demonstrated that the Glasgow coma score (GCS) at admission has a significant correlation with the two-week outcome. A score of 5 or less was associated with $85 \%$ poor outcome (defined as death or persistent coma), whereas a score of $6-8$ had a $47 \%$ chance of poor outcome. In this study it was found that the initial Glasgow coma score was an independent predictor of coma outcome. Whereas the poor prognosis with an initial Glasgow coma score of $3 \sim 5$ was $85 \%$, it rose dramatically to $47 \%$ with a Glasgow coma score of $6 \sim 8$. This correlation was also observed in our study: Glasgow Coma Score 3 5 had 59.67\% mortality and Glasgow Coma Score $6 \sim 8$ had $30.91 \%$ mortality. In another study comparing the predictive value of different scoring systems in relation to outcome, the maximum GCS was found to be the best predictor of outcome ${ }^{18}$.

Findings from the APACHE III study, which was a large-scale multicentre US study (16000 patients) showed that GCS on admission in the intensive care unit had a highly significant but non-linear relationship with subsequent outcome in patients of non-traumatic coma'. The maximum discriminatory ability of the score is in the high or low ranges of the scale. The prognostic discrimination in the middle ranges of the GCS (7-10) is less accurate?

We conclude that depth of coma as reflected by the initial Glasgow coma score is a reliable predictor of outcome in comatosed patients. Higher the Glasgow coma score at presentation, better is the prognosis.

\section{References:}

1. Olsen W H, Brumback J, Gascon G. Handbook of Symptom Oriented Neurology. Chicago Yearbook Medical Publications, Inc. 1989:190-203.

2. Lausen A A H. In Edwards C R W, Bouchier I A D, Haslett C, Chilbers Er. Davidson's Principals and Practice of Medicine. $19^{\text {th }}$ Edition. Edinburgh. Churchill Livingstone 2000.

3. Juarez V J, I yons M. Interrator reliability of Glasgow Coma Scale. J Neurosci Nurs 1995; 27(5): 283-6.

4. Menegazzi J J, Davis E A, Parris P M, Sucov Ann Neurol. Reliability of the Glasgow Coma Scale when used by the Emergency Physicians. J Trauma 1993; 34: 46-8

5. Newton C R, Kirkham F J, Johnston B, Marsh K. Interobserver agreement of coma scales and brain stem signs in non-traumatic coma. Dev Med Child Neurol 1995; 37: 807-16.

6. Sacco R L, Vangool R, Mohr J P, Hauser W A. Non-traumatic coma: Glasgow coma score and coma etiology as predictors of two week outcome. Arch Neurol 1990; 47: 1181-4.

7. Barsic B, Marton E, Himbele J, Ravlic Z. Evaluation of the Glasgow coma scale in critically ill infectious disease patients. Infection 1996; 24: 297-300.

8. Edgren E, Hedstrand U, Kelsey S, Sutton-Tyrrel K, Safar P. Assessment of neurological prognosis in comatose survivors of cardiac arrest. BRCT1 study group. Lancet 1994; 343: 1055-9.

9. Bastos P, Sun ' , Wagner O P, Wu A W, Knaus W A. Glasgow coma score in the evaluation of outcome in the intensive care unit findings from the APACHE II study. Crit Care Med 1993, 21 1459-65.

10. Gotoh O, Tamura A, Yasui, N, Manaka S, Suzuki A, Niheih et al. Japan coma scale in the prediction of outcome after early surgery for subarachnoid hemorrhage. No To Shinkei 1995; 47: 49-55.

11. Wilairtana P, Looareesuwan S. APACHE II scoring for predicting outcome in cerebral malaria. J Trop Med Hyg 1995; 98(4): 256-60.

12. Fery-Lemonnier E, Landais B, Loirat D, Klienknecht D, Brivet F. Evaluation of severity scoring systems in ICUs - translation, conversion, definition ambiguities as a source of interobsever variability in APACHE II, SAPS and OSF. Intensive Care Med 1995; 21(4): 356-60.

13. Johnston A J, Lohlum J, Miller J D, McInto:h C A, Gregori A, Brown $\mathrm{R}$ et al. A comparison of the Glasgow coma scale and the Sweedish reaction level scale. Brain Inj 1993; 7: 501-6.

14. Levy D E, Bates D, Coronna J I. Prognosis in non-traumatic coma. Ann Intern Med 1981; 94: 231.

15. Bates D. Defining prognosis in Medical coma. J Neurol Neurosurg Psychiatry 1991; 54: 557-61.

16. Nadeem M A, Irfan $\mathrm{K}$, Waheed $\mathrm{Kh} \mathrm{A} \mathrm{I,} \mathrm{Waseem} \mathrm{T,} \mathrm{Khan} \mathrm{A} \mathrm{H}$ Structural CNS diseases (non-traumatic) causing coma and their prognostic significance. P J Neurol 2000; 6(1-2): 8-12.

17. Irfan K, Nadeem M A, Waheed Kh A I, Waseem T, Khan A H. The etiology of non-traumatic (Medical) coma. Annals of King Edward Medical College Lahore 2000; 6(3): 285-88.

18. Hartley C, Cozns A, Mendalow A D, Stevenson J C. The APACHE II scoring system in neurosurgical patients: A comparison with simple Glasgow coma scoring. Br J Neurosurg 1995; 9: 179-87. 